\title{
Glasgow Coma Scale Versus Full Outline of UnResponsiveness Scale for Prediction of Outcomes in Patients with Traumatic Brain Injury in the Intensive Care Unit
}

\author{
Elham SEPAHVAND ${ }^{1}$, Rostam JALALI ${ }^{2}$, Maryam MIRZAEI ${ }^{3}$, Farzad EBRAHIMZADEH ${ }^{4}$, Mahnaz AHMADI $^{2}$, \\ Esmail AMRAII ${ }^{4}$
}

'University of Social Welfare and Rehabilitation Sciences, PhD Student of nursing, Tehran, Iran

${ }^{2}$ Kermanshah University of Medical Sciences, Department of Nursing, Kermanshah, Iran

${ }^{3}$ Jahrom University of Medical Sciences, Department of Nursing, Jahrom, Iran

${ }^{4}$ Lorestan University of Medical Sciences, Department of Paramedic, Khorramabad, Iran

\section{ABSTRACT}

AIM: Glasgow Coma Scale (GCS) is the most applied tool for classifying intensity of coma and predicting patient outcomes with traumatic brain injuries. The present study was conducted with the aim of comparing two criteria of Full Outline of UnResponsiveness (FOUR) scale and GCS in predicting prognosis in patients with traumatic brain injuries.

MATERIAL and METHODS: In this prospective study, 198 patients with traumatic brain injuries were investigated. FOUR and GCS criteria for each patient were determined by four well-educated nurses. The area under receiver operating characteristic (ROC) curve was determined for in-hospital mortality outcomes.

RESULTS: Of all patients, $65.2 \%$ survived and $34.8 \%$ died, and FOUR had correctly predicted $82 \%$ of them. FOUR had 0.76 sensitivity and GCS had a sensitivity 0.85 . Mean scores for mortality and survival rates were $4.59 \pm 2.36$ and $10.71 \pm 2.24$ in GCS, and $3.15 \pm 3.52$ and $12.77 \pm 2.43$ in FOUR, respectively. The area under ROC curve was 0.961 for FOUR and 0.928 for GCS. The area under the curve was high for FOUR in scores 6 and 7, and for GCS in scores 5 and 6.

CONCLUSION: FOUR score is a valuable, sensitive and specific diagnostic criterion for predicting outcomes in patients with traumatic brain injuries.

KEYWORDS: Traumatic brain injuries, coma, Full outline of unresponsiveness, Glasgow coma scale

\section{INTRODUCTION}

$\mathrm{T}$ Iraumatic brain injuries are a major cause of mortality and severe disability worldwide (19). On average, 1.5 million people die due to traumatic brain injuries annually, and millions require emergency medical treatment after a traumatic brain impact (12). In the United States alone, this type of injury leads to 290,000 hospitalization cases, 51,000 deaths, and 80,000 injuries in survivors (19). Initial assessment of intensity of injury in patients with traumatic brain injuries is the primary guide for medical treatment and contributes to predicting prognosis of trauma (13). Consciousness is a state of general awareness of oneself and the environment and includes the ability to orient towards new stimuli (18). Coma, or any other changes in the state of consciousness predicts outcomes and are therefore a vital clinical parameter (3). Despite technological advances, clinical assessment is still a diagnostic key to minor changes in patients' state of consciousness and the basis for management of neurology 
patients (18). Assessment of consciousness level is a vital part of basic nursing skills. Following correct assessment, the nurse is enabled to identify neurological changes in patients, and contact medical team to begin emergency actions to improve survival outcomes (4). To provide quality patient care, nurses should be able to correctly assess the patient (19). Assessment of coma and damage to consciousness level is essential in monitoring neurology patients in the special care unit (12).

It is essential to have a standard scale for measurement and assessment of consciousness level. Moreover, prognosis can be determined according to a standard scale for assessment of consciousness level (21). There are several methods for assessing impaired consciousness in brain accidents, including the NIHSS scale (National Institute of Health Stroke Scale) (which involves: assessment of consciousness, eye movement ability, visual field, power in lips and facial muscles, lips ataxia, sensory function, aphasia, dysarthria, and patient's impaired recognition and attention and only one of these items assesses level of consciousness. Thus, a precise assessment of patient's consciousness in brain accident cannot be performed) (3), Revised Trauma Scale (RTS), motor, verbal, abdominal, respiratory, circulation scale (CRAMS), Trauma Grading System (TRISS), APACHE II, Ranko scale, Rador scale, and GCS (Glasgow Coma Scale) $(11,20)$. Among these trauma severity grading systems, GCS that combines above systems has become a gold standard (19). Assessment of severity of injury is essential in clinical management of patients following brain trauma, and design of clinical trials aims to determine new treatments (7).

GCS is a practical tool for classification of intensity of coma in patients with traumatic brain injuries, even though reliability of GCS in predicting patients' outcome is unsatisfactory (19). Incomplete assessment of verbal response in intubated patients and inability to test brain stem reflexes are among failures of GCS (18). The gold standard for detecting signs of consciousness is still behavioral assessment with detailed bedside neurological assessment that can be difficult in intensive care unit (ICU), e.g. presence of orotracheal intubation, tracheotomy or motor impairment (22). Motor response capability is often affected by neuromuscular blocking agents, tranquilizers, or damages to spinal cord, and since the motor response scores the highest in GCS, its ability to correctly assess the patient is therefore impaired. Brain stem reflexes facilitate assessment of midbrain, pons and medulla oblongata functioning (18). This scale assesses various levels of damage to brain stem, and provides more accurate clinical prognosis for patient outcomes (5). Meanwhile, these are not examined in GCS scale. Impaired brain stem reflexes due to brain injuries alter respiratory pattern, and the need for mechanical ventilation can reflect intensity of coma (20). Therefore, neurological changes are not correctly and accurately assessed in GCS scale (5). In recent decades, different alternative scales to GCS have been proposed, which have not found widespread acceptability because of complexity of use (6). Full Outline of UnResponsiveness (FOUR) scale was proposed by Wijdicks et al. for assessment of neurology patients with traumatic and non-traumatic impairments of the central nervous system. FOUR scale consists of 4 items, including: eye response, motor response, brain stem reflexes, and respiratory condition, each scoring from 0 to 4 (Appendix) (5). As opposed to GCS, eye response in FOUR, as well as open eyes, also assesses their voluntary movements, which distinguishes between vegetative state of patients and minor impaired level of consciousness $(10,18)$. Accurate assessment of consciousness level is a major challenge for clinical nurses. Accordingly, it is essential for nurses to have a scale for measuring level of consciousness, so that they can constantly be aware of changes in patients' level of consciousness, and also have the right communication with medical team, using a standard and purposive tool. Thus, the researcher intended to compare FOUR scale and GCS in predicting prognosis of patients with traumatic brain injuries.

\section{- MATERIAL and METHODS}

In this prospective study, 198 ICU patients with traumatic brain injuries of the major teaching hospital of Khorramabad (Shohada Hospital) from January 2012 to May 2013 were investigated. Study inclusion criteria included over 18 years of age, survival 24 hours after admission to ICU, no intake of tranquilizers or neuromuscular blocking agents, no history of neuromuscular diseases, and no family or hereditary history of neuromuscular diseases (9). To collect data, daily observation and assessments of patients with FOUR and GCS scales were used. Three nurses tested the FOUR score and the GCS. Subsequently, raters were provided with a one-page handout written instruction describing both the FOUR score and the GCS and were asked to grade a few patients using both the GCS and the FOUR score scale. Interrater reliabilities for FOUR score and GCS were 0.98 and 0.96 , respectively. In the second 24 hours following admission to ICU, patients were assessed daily by both FOUR and GCS scales, and scores obtained for each scale were recorded. To avoid prejudice, patients were divided into groups $A$ and $B$, and the first patient in group $A$ was assessed first with FOUR and the second patient, first with GCS. The cut-off point for FOUR was considered 6 , and for GCS 5 . Scores of 6 or less in FOUR and 5 or less in GCS were considered expected death-risk (14). The four score showed high reliability after translation into Persian (20). Assessment of patients' survival or mortality continued until their discharge or death. Finally, collected data were analyzed using SPSS-18 software. Descriptive statistics were used to analyze data, including: mean, standard deviation, and frequency percentage. To determine the relationship between dependent and independent variables, Chi-square, statistics of both methods (and relevant confidence intervals) were used, receiver operating characteristic (ROC) curve was plotted, and areas under curve for both methods were compared. ROC curve was plotted for every cut-off point. Significance level was set at 0.05 .

\section{- RESULTS}

In this study, 198 patients with mean age $40.88 \pm 17.7$ years, and age range from 16 years to 80 years were assessed. $80.8 \%$ of patients were male, and $19.2 \%$ were female. Among 
all patients with traumatic brain injuries, $80(40 \%)$ had epidural hematoma, 40 (20\%) had subdural hematoma, $5(2 \%)$ had subarachnoid hematoma, and $73(36 \%)$ had hematoma in brain tissue. Of all patients, $65.2 \%$ survived, and $34.8 \%$ died, and FOUR scale correctly predicted $82 \%$ of them (Figure $1 A, B)$. Sensitivity of FOUR was 0.76 , and that of GCS was 0.85 (Table I). Mean scores for mortality and survival rates were $4.59 \pm 2.36$ and $10.71 \pm 2.24$ in GCS, and $3.15 \pm 3.52$ and

Table I: Diagnostic Value of FOUR and GCS in Predicting Death

\begin{tabular}{lcc}
\hline Scale/indicator & FOUR scale & $\begin{array}{c}\text { Glasgow coma } \\
\text { scale }\end{array}$ \\
\hline Sensitivity & $76 \%$ & $85 \%$ \\
\hline Specificity & $90 \%$ & $83 \%$ \\
\hline Positive prediction value & $83 \%$ & $64 \%$ \\
\hline Negative prediction value & $86 \%$ & $94 \%$ \\
\hline Rigor & $85 \%$ & $83 \%$ \\
\hline Likelihood ratio & 7.6 & 5 \\
\hline
\end{tabular}

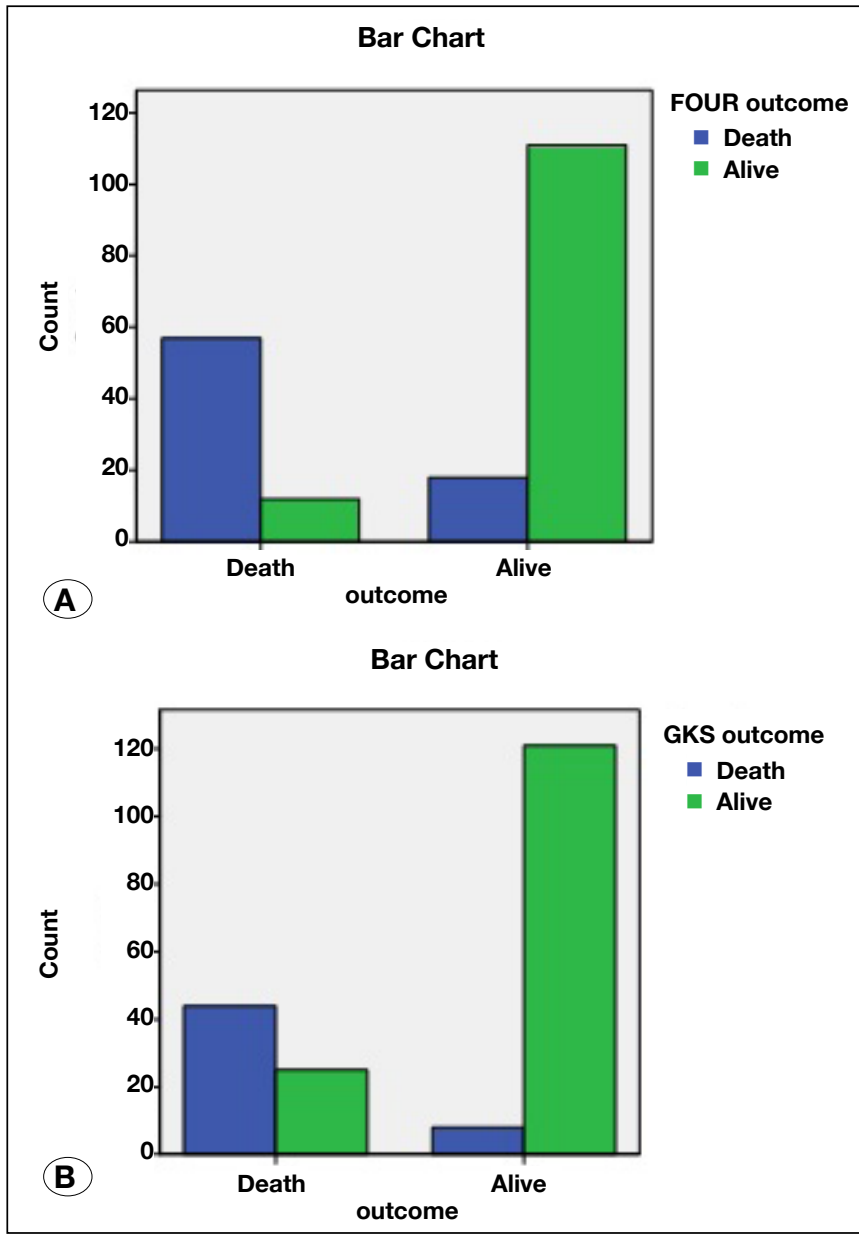

Figure 1: Prediction of survival and death outcomes by FOUR (A) and GCS (B) scales.
$12.77 \pm 2.43$ in FOUR, respectively. T-test showed a significant difference between survival and death of patients in the FOUR scale $(p=0.000)$, but the difference in GCS scale was insignificant $(p=0.542)$. The area under $R O C$ curve was 0.961 in FOUR, and 0.928 in GCS (Figure 2). The area under curve was high for FOUR in scores 6 and 7, and high for GCS in scores 5 and 6 . The distribution of all ratings of the FOUR score and the GCS score is shown in Figures 3 and 4.

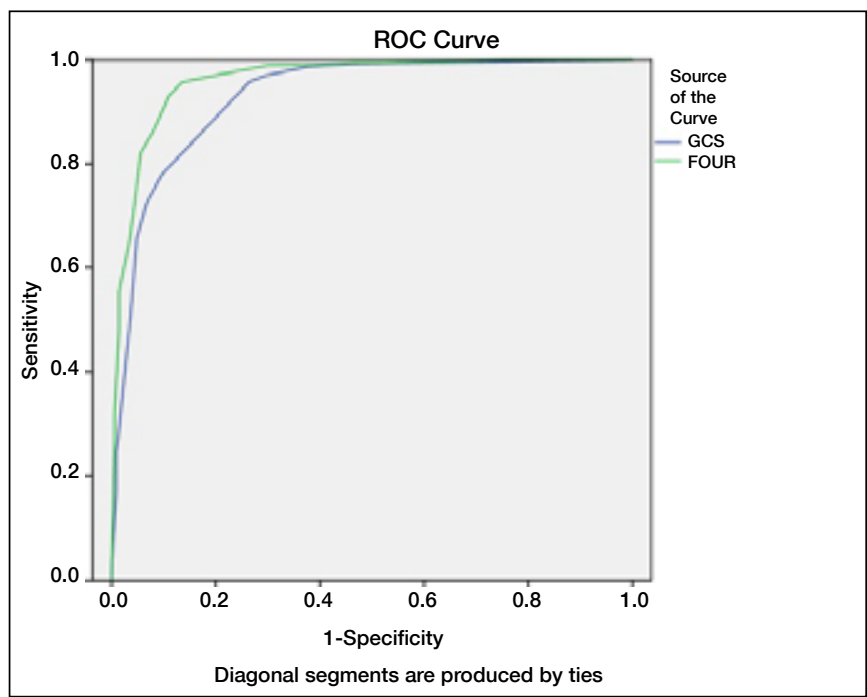

Figure 2: Area under curve for FOUR and GCS scales.

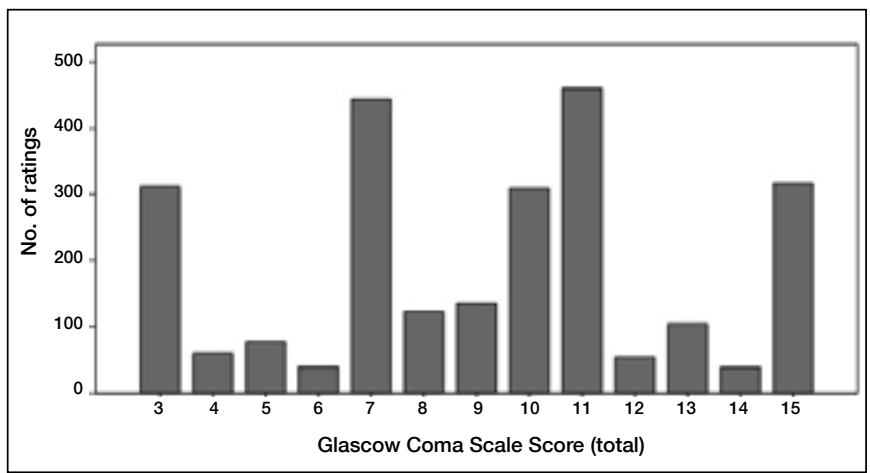

Figure 3: Distribution of total Glasgow Coma Scale Score.

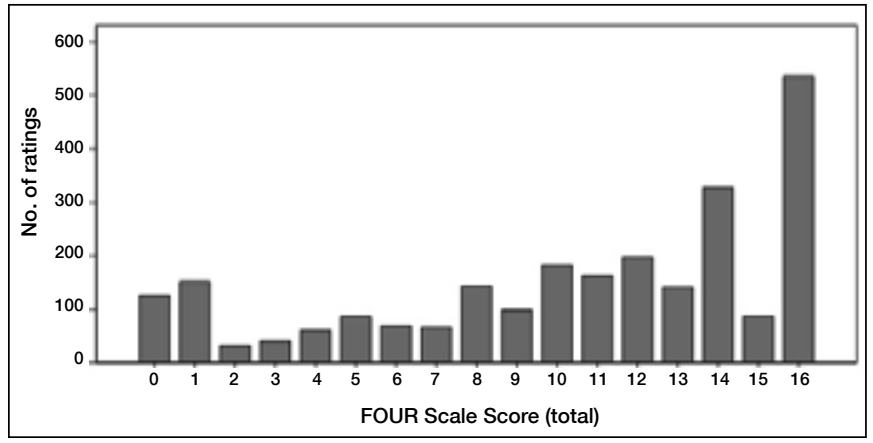

Figure 4: Distribution of total FOUR scale scores. 


\section{DISCUSSION}

Neurological impairments are a major challenge for ICU patients and those with neurological damage. In line with other studies, the present study results indicate that FOUR is a correct predictor of mortality in ICU patients with traumatic brain injuries $(1,14,19)$. FOUR is easy to use, and provides better information, particularly in intubated patients, and shows depth of coma in greater details compared to GCS, since it assesses brain stem reflexes and respiratory pattern (18). Considering failures of GCS in assessing patients' level of consciousness, Sadaka et al. recommended the FOUR scale (19). FOUR better classifies level of consciousness damage in emergency patients $(15,16)$, cardiac arrest patients (8), and ICU patients (12) compared to other scales. Furthermore, FOUR has greater validity and reliability among nurses, neurologists, and doctors $(5,6,11,20)$. In the present study, FOUR showed high predicting value for in-hospital mortality, which agreed with Sadaka study (19). Mercy et al. study indicated minor differences between FOUR and GCS scales in prediction value for in-hospital deaths, and argued that, FOUR and GCS are equally able to predict mortality in neurological patients (18).

In the present study, area under ROC curve showed that both FOUR and GCS were excellent predictors of in-hospital mortality, which concurs with other studies $(2,14,17)$. In the present study, likelihood ratio was higher in FOUR scale than in GCS, which indicates greater ability of this scale in better identifying patient outcomes (17). This scale also assesses neurological status of patients with greater accuracy. High sensitivity of FOUR scale in clinical assessment has eased treatment and monitoring of patients for the medical team.

The originator of FOUR considers cut-off point of 9 valid for in-hospital mortality, while cut-off point in the present study was 6-7, and 10, in Akavipat study (1). The difference could be due to patient's conditions and the environment where the patient is hospitalized.

FOUR assesses locked-in syndrome more accurately and faster by examining voluntary eye movements (18). This is a valid and practical scale, which can be used after simple training of medical team, and has a high degree of practical confidence, and high rigor as a diagnostic indicator in determining level of consciousness (10). Yet, due to global acceptability, GCS is still used to assess patients with neurological injuries (5).

\section{CONCLUSION}

According to the present study results, FOUR is a new, valuable, sensitive and specific scale for predicting outcomes in patients with traumatic brain injuries. Using brain stem reflexes and respiratory pattern, this scale provides an accurate and correct assessment of patients in coma, and has the ability to assess minor changes in neurological status of patient. Therefore, FOUR is recommended as a tool for assessment of neurological patients with changes in consciousness levels. It is also recommended that medical team be trained to use this scale beforehand.

\section{ACKNOWLEDGEMENTS}

We thank all personnel in the Intensive Care Unit of Shohada Hospital in Khorramabad for their assistance in this study. Also, we wish to thank the Farzan Institute for Research and Technology for their technical assistance.

\section{REFERENCES}

1. Akavipat $P$, Sookplung $P$, Kaewsingha $P$, Maunsaiyat $P$ : Prediction of discharge outcome with the Full Outline of UnResponsiveness (FOUR) score in neurosurgical patients. Acta Med Okayama 65: 205-210, 2011

2. Bruno M, Ledox D, Lambermont B, Damas F, Schnakers C, Vanhaudenhuyse A, Gosseries O, Laureys S: Comparison of the full outline of unresponsiveness and glasgow liege scale/ Glasgow coma scale in an intensive care unit population. Neurocrit Care 15:447-453, 2011

3. Carolei A, Sacco S: The FOUR score: A reliable instrument to assess the comatose stroke patient. Eur Neural 63:370-371, 2010

4. Chan M, Mattar I, Taylor B J: Investigating factors that have an impact on nurses 'performance of patients' conscious level assessment: a systematic review. J Nursing Manag 21: 31-46, 2013

5. Chen B, Grothe $\mathrm{CH}$, Schaller $\mathrm{K}$ : Validation of a new neurological score (FOUR Score) in the assessment of neurosurgical patients with severely impaired consciousness. Acta Neurochir 155:2133-2139, 2013

6. Fischer M, Reugg S, Czaplinski A, Strohmeier M, Lehmann A, Tschan F, Hunziker P, Marsch S: Interrater reliability of the full outline of unresponsiveness score and Glasgow coma scale in critically ill patients: A prospective observational study. Neurocrit Care 14: R64, 2010

7. Foreman BP, Caesar R, Parks J, Madden CH, Gentilello L M, Shafi SH, Carlile MC, Harper CR, Diaz-Arrastia RR: Usefulness of the abbreviated injury score and the injury severity score in comparison to the Glasgow Coma Scale in predicting outcome after traumatic brain injury. J Trauma 62:946 -950, 2007

8. Fugate JE, Rabinstein AA, Claassen DO, White RD, Wijdicks EFM: The FOUR score predicts outcome in patients after cardiac arrest. Neurocrit Care 13: 205- 210, 2010

9. Ghayeghran A, Bakhshayesh Eghbali B, Heydarzadeh A: Survey the correlation between $F$ wave persistence and level of consciousness in stroke patients. Journal of Guilan University of Medical Sciences 72:70-76, 2009

10. Idrovo Freire L: The FOUR score: Is it just another new coma scale? Intern Emerg Med 7: 203-204, 2012

11. Idrovo L, Fuentes B, Medina J, Gabaldón L, Ruiz-Ares G, Abenza MJ, Aguilar-Amat MJ, Martínez-Sánchez P, Rodríguez L, Cazorla R, Martínez M, Tafur A, Wijdicks EF, Diez-Tejedor E: Validation of the FOUR score in acute stroke, An Interobserver validity study. Eur Neurol 63: 364-369, 2010

12. Iyer VN, Mandrekar JN, Danielson RD, Zubkov AY, Elmer JL, Wijdicks EF: Validity of the FOUR score coma scale in the medical intensive care unit. Mayo Clin Proc 84: 694-701, 2009 
13. Izadi Avanji F, Fakharian S, Masoodi Alavi N: Outcome of factors related to traumatic brain injuries among the patients hospitalized in intensive care unit. Journal of Kashan University of Medical Sciences 14: 112-119, 2010

14. Jalali R, Rezaei M: A comparison of the Glasgow Coma Scale (GCS) score with Full Outline of Unresponsiveness (FOUR) scale to predict patients' traumatic brain injury outcomes in intensive care units. Critic Care Res Pract Article ID 289803: 4 pages, 2014. Available from: http://dx.doi. org/10.1155/2014/289803

15. Kevric J, Jelinek GA, Knott J, Weiland TJ: Validation of the Full Outline of Unresponsiveness (FOUR) scale for conscious state in the emergency department: Comparison against the Glasgow Coma Scale. Emerg Med J 28: 486-490, 2011

16. Ledoux D, Bruno M, Jonlet S, Choi P, Schnakers C, Damas F, Lambermont B, Damas P, Laureys S: Full outline of unresponsiveness compared with Glasgow Coma Scale assessment and outcome prediction in coma. Critical Care 13: P107, 2009

17. Marcati E, Ricci S, Casalena A, Toni D, Carolei A, Sacco S: Validation of the Italian version of a new coma scale: The FOUR score. Intern Emerg Med 7:145-152, 2012

18. Mercy SA, Thakur R, Yaddanapudi S, Bhagat H: Can FOUR Score replace GCS for assessing neurological status of critically ill patients - An Indian Study. Nurs Midwifery Res J 9:63-72, 2013

19. Sadaka F, Patel D, Lakshmanan R: The FOUR score predicts outcome in patients after traumatic brain injury. Neurocrit Care 16:95-101, 2012

20. Tadrisi SD, Bahari N, Ebadi A, Madani SJ: Validity and reliability of coma scale (Four Score) in adult patient hospitalized in critical care units. Critic Care Nurs 5: 95 - 100, 2012

21. Tindall SC: Level of consciousness. In: Walker HK, Hall WD, Hurst JW (eds). Clinical Methods: The History Physical and Laboratory Examinations. $3^{\text {rd }}$ ed. Boston: Butterworths, 1990: 371-382

22. Weiss N, Tadie JM, Faugeras F, Diehl JL, Fagon JY, Guerot E: Can fast-component of nystagmus on caloric vestibulo-ocular responses predict emergence from vegetative state in ICU? J Neurol 259: 70-76, 2012

\section{APPENDIX}

Full Outline of UnResponsiveness (FOUR)

A.2.1. Eye Response. One has the following:

4 = eyelids open or opened, tracking, or blinking to command

3 = eyelids open but not to tracking

2 = eyelids closed but open to loud voice

1 = eyelids closed but open to pain

$0=$ eyelids remaining closed with pain stimuli.

A.2.2. Motor Response. One has the following:

$4=$ thumbs up, fist, or peace sign

$3=$ localizing to pain

2 = flexion response to pain

$1=$ extension response

$0=$ no response to pain or generalized myoclonus status.

A.2.3. Brain Stem Reflexes. One has the following: $4=$ pupil and corneal reflexes present

$3=$ one pupil wide and fixed

2 = pupil or corneal reflexes absent

$1=$ pupil and corneal reflexes absent

0 = absent pupil, corneal, or cough reflex.

A.2.4. Respiration. One has the following:

4 = regular breathing pattern

3 = Cheyne-Stokes breathing pattern

2 = irregular breathing

$1=$ triggering ventilator or breathing above ventilator rate

0 = apnea or breathes at ventilator rate. 